\title{
Deleterious mutation in the $F Y B$ gene is associated with congenital autosomal recessive small-platelet thrombocytopenia
}

\author{
C. LEVIN $^{1,2}$, A. KOREN ${ }^{1,2}$, E. PRETORIUS ${ }^{3}$, N. ROSENBERG ${ }^{4}$, \\ B. SHENKMAN ${ }^{4}$, H. HAUSCHNER ${ }^{4}$, L. ZALMAN ${ }^{5}$, M. KHAYAT ${ }^{6}$, I. SALAMA ${ }^{7}$, \\ O. ELPELEG ${ }^{8}$, and S. SHALEV ${ }^{2,6}$
}

\author{
${ }^{1}$ Pediatric Hematology Unit and Pediatric Department B, Emek Medical Center, Afula; \\ ${ }^{2}$ The Ruth and Baruch Rappaport School of Medicine, Technion, Israel Institute of \\ Technology, Haifa, Israel; \\ ${ }^{3}$ Department of Anatomy, Faculty of Health Sciences, School of Medicine of the \\ University of Pretoria, Gauteng, South Africa; \\ ${ }^{4}$ Institute of Thrombosis and Hemostasis, Chaim Sheba Medical Center, Tel Hashomer and \\ Sackler Faculty of Medicine, Tel-Aviv University, Tel-Aviv; \\ ${ }^{5}$ Hematology Laboratory, Emek Medical Center; \\ ${ }^{6}$ Genetic Institute, Emek Medical Center; \\ ${ }^{7}$ Clalit Health Services, Afula; and \\ ${ }^{8}$ Monique and Jacques Roboh Department of Genetic Research, Hadassah, Hebrew \\ University Medical Center, Jerusalem, Israe
}

\section{Summary}

Background: The $F Y B$ gene encodes adhesion and degranulation-promoting adaptor protein (ADAP), a hematopoietic-specific protein involved in platelet activation, cell motility and proliferation, and integrin-mediated cell adhesion. No ADAP-related diseases have been described in humans, but ADAP-deficient mice have mild thrombocytopenia and increased rebleeding from tail wounds.

Patients and methods: We studied a previously reported family of five children from two consanguineous sibships of Arab Christian descent affected with a novel autosomal recessive bleeding disorder with small-platelet thrombocytopenia. Homozygosity mapping and exome sequencing were used to identify the genetic lesion causing the disease phenotype on chromosome 5. Bone-marrow morphology and platelet function were analyzed. Platelets were characterized by scanning electron microscopy.

Results: We identified a homozygous deleterious nonsense mutation, c.393G>A, in $F Y B$. A reduced percentage of mature megakaryocytes was found in the bone marrow. Patients' platelets showed increased basal expression of P-selectin and PAC-1, and reduced increments of activation markers after stimulation with ADP, as detected by flow cytometry; they also showed reduced pseudopodium formation and the presence of trapped platelets between the fibrin fibers after thrombin addition, as observed on scanning electron microscopy.

Conclusions: This is the first report of a disease caused by an FYB defect in humans, manifested by remarkable small-platelet thrombocytopenia and a significant bleeding tendency. The described phenotype shows ADAP to be important for normal platelet production, morphologic changes, and function. It is suggested that mutation analysis of this gene be included in the diagnosis of inherited thrombocytopenia. 
Keywords: adaptor proteins, signal transducing; blood platelet disorders; humans; inherited blood coagulation disorders; mutation.

\section{Introduction}

A growing number of inherited thrombocytopenia conditions have been recently recognized and characterized, with the diagnosis being based on inheritance pattern, platelet morphology and size, bone-marrow morphology, and associated clinical features [1-3]. The underlying genetic defects are often known, but their molecular mechanisms remain unresolved [4].

Prior to our description of congenital autosomal recessive small-platelet thrombocytopenia (CARST) [5], only two inherited diseases were known to cause small platelets: WiskottAldrich syndrome (WAS) and X-linked thrombocytopenia, caused by mutations in the WAS gene [6]. Here, we report a hematologic and molecular investigation of patients with CARST [5].

\section{Materials and methods}

All laboratory and DNA analyses of the patients, family members and controls were performed after written, informed consent and Ethical Review Board of Emek Medical Center approval had been obtained. Details of patients' ethnicity and religion are routinely used in our service, facilitating genetic diagnoses implemented under the regulations of the Israel genetic information law [7].

\section{Genomic DNA isolation}

Genomic DNA was extracted with the FlexiGene DNA kit (Qiagen, Hilden, Germany), according to the manufacturer's instructions.

\section{Homozygosity mapping}

Patients' DNA samples were analyzed by use of the Illumina OmniExpress BeadChip, which contains 730525 single-nucleotide polymorphism (SNP) markers, and Illumina genomestudio software (Illumina, San Diego, CA, USA). The analysis report included the 20 largest homozygous regions observed, the genes found within these regions, and the On-line Mendelian Inheritance in Man phenotypes, where available.

\section{Whole-exome analysis}

Exonic sequences were enriched in patient 4's DNA sample with the SureSelect Human All Exon $50 \mathrm{Mb}$ kit (Agilent Technologies, Santa Clara, CA, USA). Sequences, consisting of 100-bp paired-end reads, were determined with HiSeq2000 (Illumina). Read alignment and variant calling were performed by the use of DNAnexus software (DNAnexus, Palo Alto, CA, USA), with the default parameters with human genome assembly hg19 (GRCh37) as reference [8]. 


\section{Sanger sequence analysis}

Sequence analysis was performed with $243 \mathrm{bp}$ of the PCR product within exon 2 of $F Y B$ that was amplified with the sense primer 5'-CAAGCCCCCGTTTCTAAAGC-3' and the antisense primer 5'-GGAGTAGGCCCAGATTTCGG-3'. Genomic DNA samples from five patients and nine healthy individuals, and cDNA from two patients and two healthy individuals, were sequenced with the Bigdye terminator kit, and analyzed with the ABI PRISM 3130xl Genetic Analyzer, according to the manufacturer's instructions (Applied Biosystems, California, USA).

\section{Whole-blood preparation for scanning electron microscopy (SEM)}

Blood was collected in citrate tubes from one control and from patients 1 and 2 . Whole blood and platelet-rich plasma (PRP) $(10 \mu \mathrm{L})$ were prepared on a glass coverslip with or without $5 \mu \mathrm{L}$ of human thrombin $\left(20 \mathrm{U} \mathrm{mL}^{-1}\right.$ in biological buffer containing $0.2 \% \mathrm{v} / \mathrm{v}$ human serum albumin), incubated at $37{ }^{\circ} \mathrm{C}$ for $10 \mathrm{~min}$, washed in $0.075 \mathrm{~m}$ phosphate-buffered saline (PBS) on a shaker $(20 \mathrm{~min})$, fixed in formaldehyde $(30 \mathrm{~min})$, washed three times in $0.075 \mathrm{~m}$ PBS (3 min), postfixed with $1 \%$ osmium tetroxide (15 min), washed in $0.075 \mathrm{~m}$ PBS (3 min), serially dehydrated in 30\%, 50\%, 70\%, 90\% and 100\% (three times) ethanol, dried with hexamethyldisilazane, mounted, coated with carbon, and examined by SEM (Zeiss ULTRA plus FEG SEM, Oberkochen, Germany). A blood clot from each individual, which formed on the coverslip and covered an area of $\sim 1 \mathrm{~cm}^{2}$, was viewed. The average number of visible platelets among fibrin fibers was calculated per $250 \mu \mathrm{m}^{2}$.

Patients' micrographs were compared with those from a healthy control and with our database of healthy individuals. The control micrographs were representative of normal samples as found in our healthy platelet database and the literature. The micrographs shown are representative of all samples.

\section{Platelet-function analyses}

Blood was collected in citrate tubes (from patients 1 and 2, a heterozygous individual, and a normal control). Studies were performed in duplicate.

Platelet adhesion was evaluated in whole blood subjected to flow at $1200 \mathrm{~s}^{-1}$ by use of a cone and platelet analyzer (CPA) (Impact-R), as previously described [9]; seven fields of $0.7 \mathrm{~mm}^{2}$ were analyzed. The control/heterozygous samples were reconstituted to $35 \times 10^{9}$ platelets $\mathrm{L}^{-1}$ for comparison with the patients' platelet counts; normal values were used as a reference [9, $10]$.

Platelet activation was measured by flow cytometry of PRP with and without $10 \mu \mathrm{m}$ ADP (Molab, Langenfeld, Germany), with the following anti-human mAbs: P-selectin CD62pphycoerythrin (PE) (BioLegend, San Diego, CA, USA), CD41-PE cyanin-7 (Beckman Coulter, Marseille, France), CD42b (Dako, Glostrup, Denmark) and PAC-1-fluorescein isothiocyanate (BD Biosciences, San Jose, CA, USA), which recognizes only the active conformation of integrin $\alpha_{\mathrm{IIb}} \beta_{3}$ [11]. At least 2000 single platelets were acquired per sample. 


\section{Study limitations}

Owing to the limited number of patients, and the small sample size in the studies performed, statistical comparison between patients and controls was not possible.

\section{Results and discussion}

We describe five individuals of Arab Christian ancestry with small-platelet thrombocytopenia with neonatal onset and an increased bleeding tendency, but with normal growth and development and an absence of infections during 17 years of follow-up. The pedigrees were consistent with autosomal recessive inheritance.

All patients' physical examinations were normal, with no associated clinical features other than eczema during infancy (three patients). Bleeding symptoms included petechial rash, mucosal and heavy menstrual bleeding, and intra-abdominal bleeding from a ruptured corpus luteum. A detailed clinical description and family pedigree have been published previously $[5]$.

Patients' complete blood counts showed a normal white blood cell count, a low platelet count $\left(5-69 \times 10^{9} \mathrm{~L}^{-1}\right)$, a low mean platelet volume (5.8-7.2 fL), and mild iron deficiency anemia that responded to iron treatment. Peripheral blood smears showed small platelets and decreased numbers, with no additional abnormalities (Fig. 1A-C).

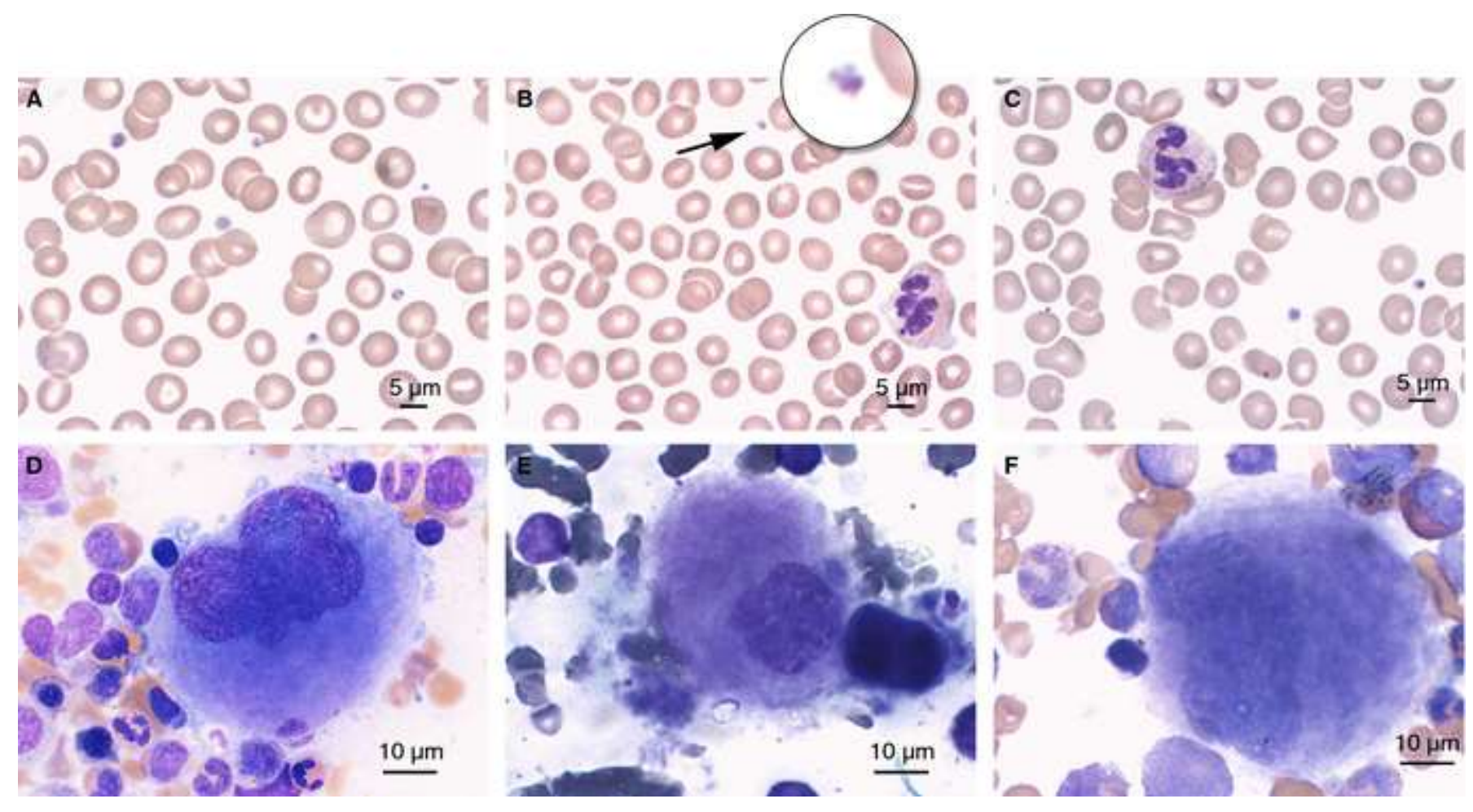

Figure 1. Peripheral blood smear and bone-marrow aspirate morphology images obtained with optical microscopy. Photomicrographs of peripheral blood smears and bone-marrow aspirates are shown after WrightGiemsa staining at high-power magnification. (A) Normal platelet number and size in a healthy control. (B, C) Decreased platelet number and small size (blood smear from patient 2); (C) is unusual, because two platelets can be seen in one field. Scale bar: $5 \mu \mathrm{m}$. (D) Bone-marrow aspirate from an unaffected control, showing a normal megakaryocyte. (E, F) Bone-marrow aspirate from patient 4, showing two representative megakaryocytes of normal size, but with reduced nuclear complexity as compared with healthy megakaryocytes. Scale bar: $10 \mu \mathrm{m}$. 
Bone-marrow aspirate from patient 4 was reviewed and compared with that from two unaffected controls (at least 30 megakaryocytes analyzed per sample); the patient's aspirate showed normal cellularity, erythroid and myeloid differentiation, and numbers and size of megakaryocytes (data not shown); however, the percentage of mature multilobulated megakaryocytes (three or more nuclear lobules) was reduced as compared with controls (15\% versus $68 \%$ ). A predominance of megakaryocytes with nuclear hyposegmentation was noted (Fig. 1D-F), suggesting abnormal maturation of megakaryocytes involved in the pathogenesis of the thrombocytopenia.

SEM analysis of healthy individual platelets showed slight pseudopodium formation, probably because of contact activation during preparation (Fig. 2A). However, no pseudopodia were observed in the patients' platelets prepared under the same conditions (Fig. 2C).
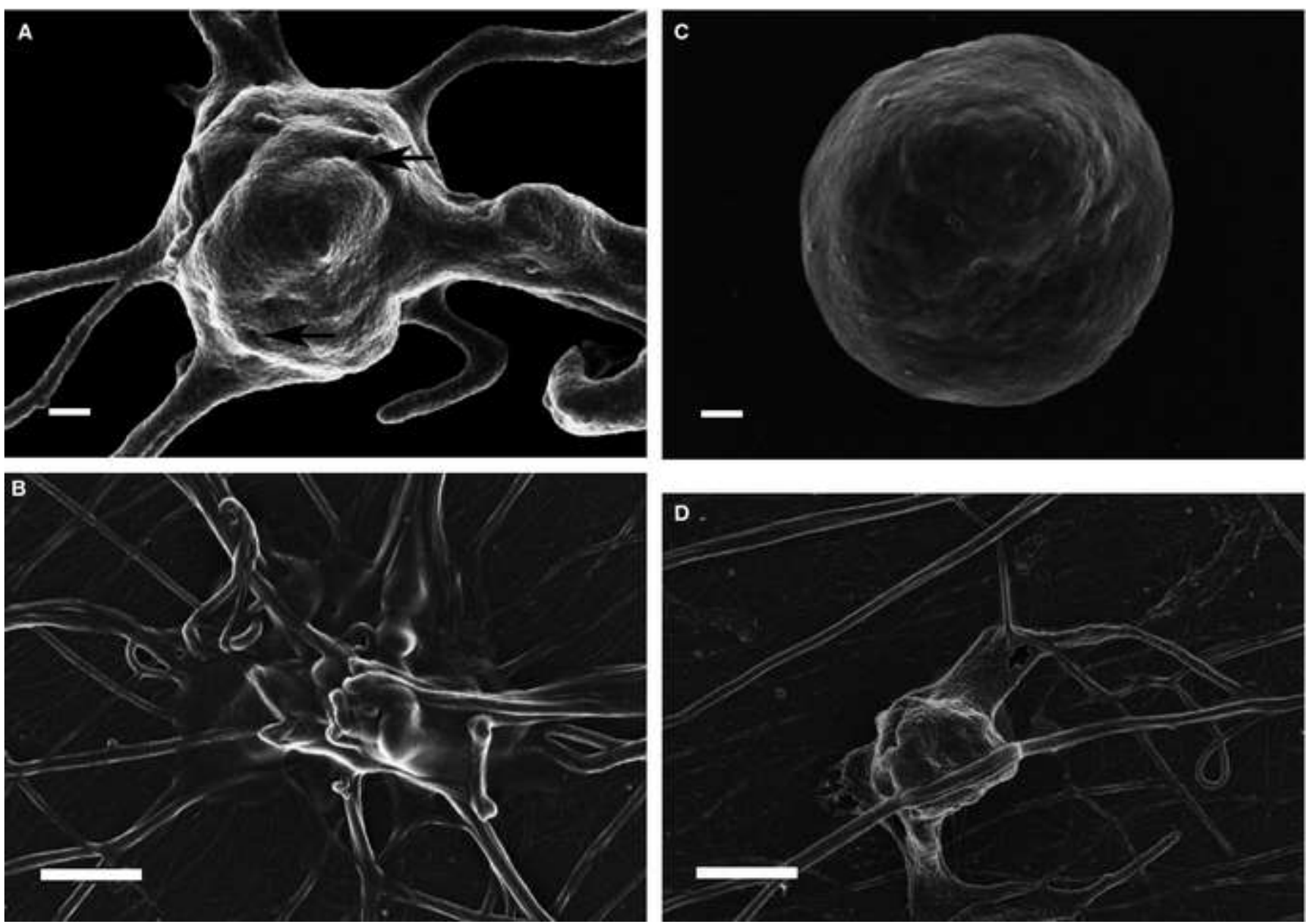

Figure 2. Scanning electron microscopy analysis of platelets and fibrin fibers in whole-blood smears. (A, B) Healthy individual's samples. (C, D) Representative micrographs from patients 1 and 2. (A) Platelet as seen in a whole-blood smear, showing slight pseudopodium formation, and open canalicular system channels, indicated with black arrows. Scale bar: $200 \mathrm{~nm}$. (B) After thrombin addition: a typical fibrin network and activated platelet spreading and merging with the fibrin fibers. Scale bar: $1 \mu \mathrm{m}$. (C) Patient 1 platelet, as seen in a wholeblood smear, with no pseudopodium formation and no prominent open canalicular system channels. Scale bar: $200 \mathrm{~nm}$. (D) Patient 2 platelet after thrombin addition, trapped in a fibrin network; platelets do not merge with the fibrin fibers or spread extensively, but stay mostly intact. Scale bar: $1 \mu \mathrm{m}$. 


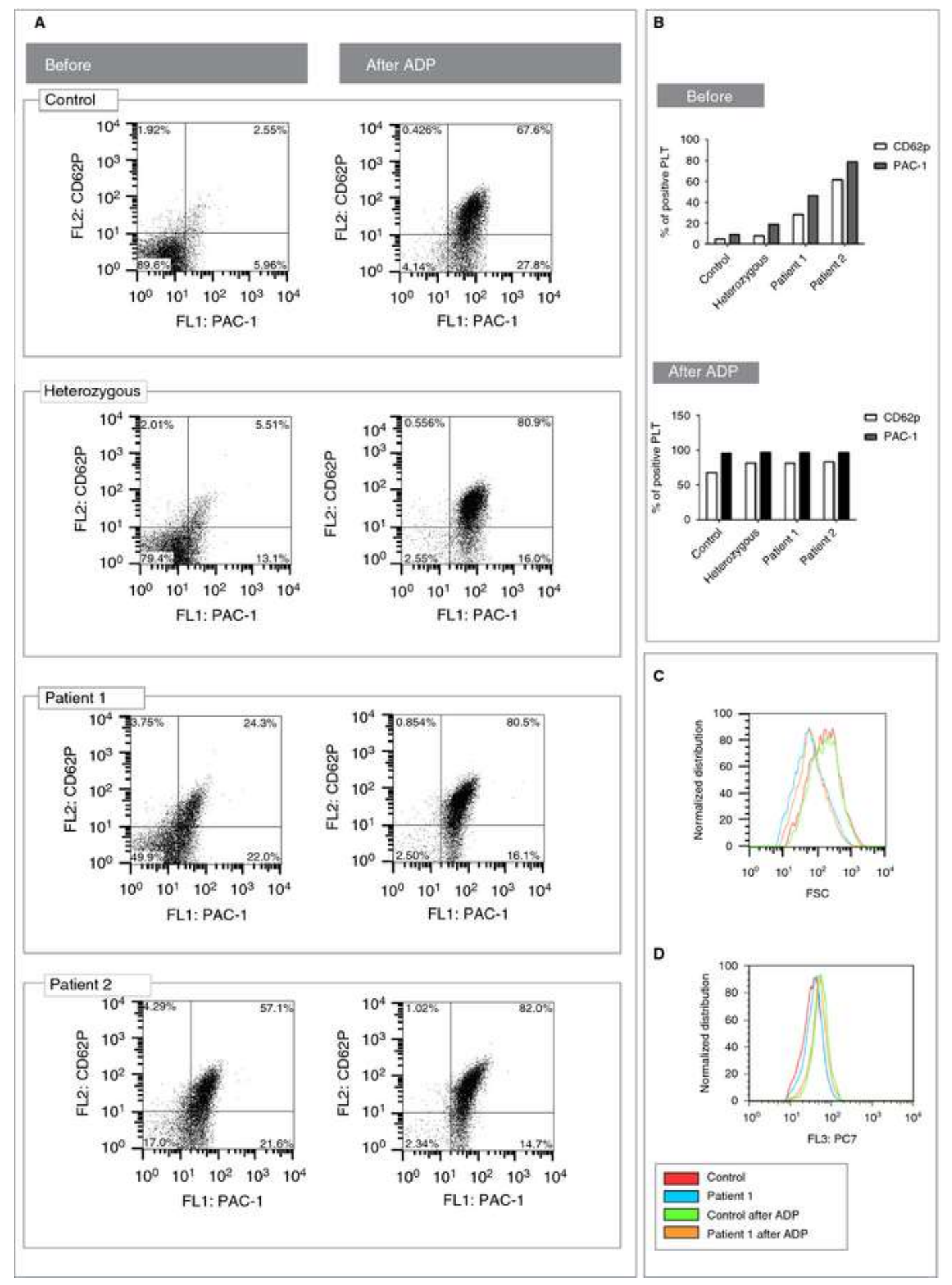

Figure 3. Platelets' basal flow cytometry and activation with ADP. Staining with anti-CD41 was used to gate on platelets. (A) Representative dot plot of CD62p versus PAC-1 fluorescence before and after stimulation with ADP. Platelets positive for both CD62p and PAC-1 are in the right upper quadrant. Increased surface expression of CD62p and PAC-1 before addition of ADP was observed on patients' platelets as compared with platelets from control and heterozygous individuals. (B) Percentages of positive platelets (CD62p and PAC-1) before and after addition of ADP. A reduced agonist-dependent increment of CD62p and PAC-1 expression was observed in patients' platelets. (C) Representative histogram of forward-scattered light (FSC) versus a normalized distribution of platelets, showing reduced platelet size in the patient as compared with a control, with no significant changes in size after addition of ADP. (D) Representative histogram of the fluorescence intensity of phycoerythrin cyanin-7 (PC7)-conjugated anti-CD41 in patient 1 and the control, before and after addition of ADP. PLT, platelets. 
When thrombin is added to healthy whole blood, a fibrin-fiber network forms. Upon activation, the platelets release their granular contents and merge with the fibrin fibers. Thus, platelets are seen extremely rarely in healthy whole blood after thrombin addition [12-15].

SEM analysis after thrombin addition in the healthy individual sample showed a typical fibrin network and an activated platelet, spreading and merging with the adjacent fibrin (Fig. 2B). In the patients' samples, persistently discoid, trapped platelets with very few pseudopodia were noted between the fibrin fibers (Fig. 2D). The average number of trapped/visible platelets per $250 \mu \mathrm{m}^{2}$ of sample was higher in the patients (five and three for patients 1 and 2 , respectively; only one visible merging - but not trapped - platelet in the control sample). This finding correlates with previous studies in adhesion and degranulation-promoting adaptor protein (ADAP)-deficient mice showing reduced formation of platelet filopodia $[16,17]$. Further analysis of platelet spreading on different matrices is warranted.

Flow-cytometry analysis of patients' platelets showed normal expression of both glycoprotein (GP)Ib-IX and integrin $\alpha_{\mathrm{IIb}} \beta_{3}$; after adjustment for platelet size, a $50 \%$ increase was found relative to controls (data not shown); the basal expression of P-selectin and PAC-1 was greater in patients than in the control or heterozygote (respectively: $28 \%$ and $62 \%$ versus $4.5 \%$ and $7.5 \%$ for P-selectin; $46 \%$ and $79 \%$ versus $8.5 \%$ and $18.6 \%$ for PAC-1) (Fig. 3A,B). Upon addition of ADP, the increment in double-positive (CD62p and PAC-1) platelets was lower in patients than in the control and the heterozygote (1.4-fold and 3.3-fold versus 26.5fold and 14.7-fold, respectively). Thus, increased clearance of activated platelets might contribute to the thrombocytopenia.

Platelet adhesion was evaluated with the CPA. Fibrinogen and von Willebrand factor interact with the polysterene surface to mediate platelet adhesion. GPIb-IX and integrin $\alpha_{\mathrm{IIb}} \beta_{3}$ are needed for platelet deposition in this system [18].

Slight decreases in aggregate size and surface coverage for patient platelets as compared with those from the control and heterozygous individuals (before and after reconstitution to $35 \times 10^{9}$ platelets $\mathrm{L}^{-1}$, respectively) were found; the values remained within the normal range, except for surface coverage in patient 1 (1.7\% as compared with $3-3.8 \%$ for the same platelet count in the control and heterozygous individuals) (Fig. 4A). Additional platelet-adhesion studies are required.

Homozygosity mapping in the five patients revealed one major shared block of homozygosity on chromosomes 5p13.2-q11.2 that was localized to Chr5:37 921 839-51 690 015. This 13.7-Mb region contained 47 predicted or known genes.

Following alignment and variant calling, we removed variants that were off-target, were read less than 8 times, were heterozygous, or were X-linked, or that were present in dbSNP132 or the in-house dbSNP. Of the six remaining variants, only one, Chr5:39202670 C>T, NM_001465 c.G393A, p.W131X, in the FYB, was located within the shared 13.7-Mb homozygous region. This mutation was not present in 60376 exome analyses deposited at Exome Aggregation Consortium (ExAC) website, Cambridge, MA (URL: http://exac.broadinstitute.org) (accessed April 2015), and segregated with the disease in the family. 


\begin{tabular}{|cccc|}
\hline A Samples & $\begin{array}{c}\text { Surface } \\
\text { coverage } \\
\%\end{array}$ & $\begin{array}{c}\text { Aggregate size } \\
\mu \mathrm{m}^{2}\end{array}$ & $\begin{array}{c}\text { Platelet count } \\
\times 10^{9} \text { per L }\end{array}$ \\
\hline $\begin{array}{c}\text { Control } \\
\text { Heterozygous }\end{array}$ & 5.8 & 63 & 225 \\
\hline Patient 1 & 1.1 & 74 & 200 \\
\hline $\begin{array}{c}\text { Patient 2 } \\
\text { Reconstituted } \\
\text { control }\end{array}$ & 3.1 & 39.5 & 37 \\
$\begin{array}{c}\text { Reconstituted } \\
\text { heterozygous }\end{array}$ & 3.8 & 54 & 33 \\
\hline Normal range & $11.72 \pm 3.1$ & $44.93 \pm 16.4$ & 35 \\
\hline
\end{tabular}

B

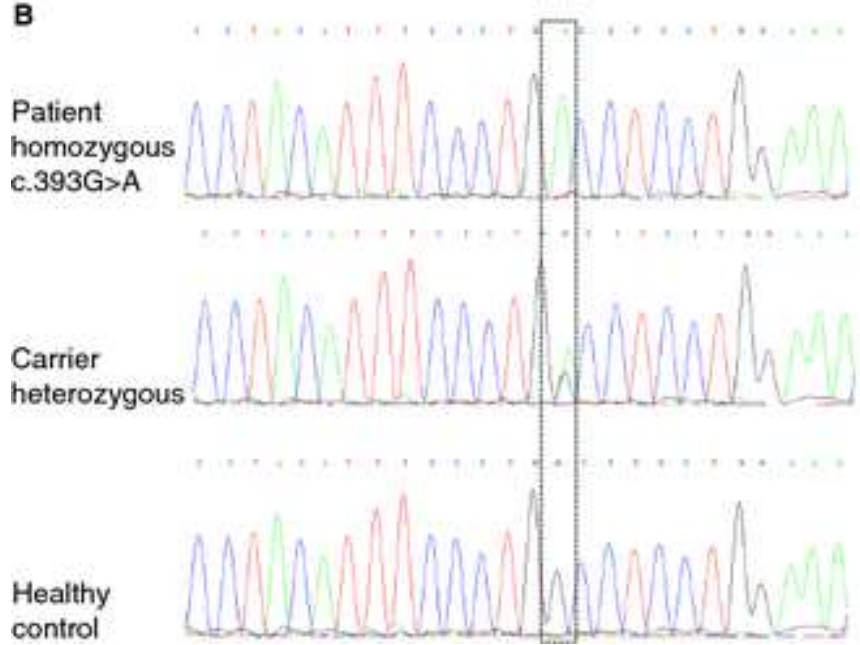

Figure 4. Platelet adhesion and DNA-sequence analyses. (A) Surface coverage and aggregate size measured with a cone and platelet analyzer. Platelet adhesion was evaluated in whole blood flowing at $1200 \mathrm{~s}^{-1}$. The data show a slightly reduced aggregate size in the patients as compared with the normal control and heterozygous individuals (before and after sample reconstruction to $35 \times 10^{9}$ platelets $\mathrm{L}^{-1}$ ) that is nevertheless still within the normal range. Surface coverage is lower in the patients than in the normal control and the heterozygote; after reconstitution of the control and heterozygous samples to $35 \times 10^{9}$ platelets $\mathrm{L}^{-1}$, the same decrease in surface coverage is observed. (B) Relevant sections of the DNA-sequence analyses of exon 2 of $F Y B$ from patient 1 (patient, homozygous), one parent (carrier, heterozygous), and a healthy control. All five patients shared the homozygous mutation c. $393 \mathrm{G}>\mathrm{A}$; the patients' parents and one brother were heterozygous for this mutation.

Sequence analysis of exon 2 of $F Y B$ revealed homozygosity for the c.393G $>$ A nonsense mutation in the five patients; the parents and one brother were heterozygous (Fig. 4B). The c.393G > A mutation was not detected in 60 healthy individuals of the same ethnic background as the patients.

The mutation is predicted to lead to premature translation termination of the encoded 829amino acid ADAP.

ADAP is a $120-130-\mathrm{kDa}$ cytosolic adaptor protein expressed by platelets, T cells, natural killer cells, myeloid cells and dendritic cells that is involved in cell motility, proliferation, activation, and cytokine production $[16,19,20]$.

ADAP was originally identified as an $\mathrm{SH} 2$-binding protein that inducibly binds lymphocyte cytosolic protein 2 (SLP76) and the src-family kinase Fyn, and is phosphorylated during the T-cell signaling cascade [20-22]. ADAP is involved in actin-cytoskeleton changes after Tcell activation [23]. The functional cooperation between Nck and ADAP mediates SLP76WAS protein interactions and actin rearrangement [24]; furthermore, ADAP is postulated to 
directly link integrins to cytoskeleton modulators, independently of SLP76 [20]. ADAP is a candidate linker between cell-membrane activation signals and intracellular events that modify actin polymerization and organization [16].

ADAP is important for platelet activation, participates in inside-out regulation of integrin $\alpha_{\mathrm{IIb}} \beta_{3}$ on platelets [16], is an essential component of $\alpha_{\mathrm{IIb}} \beta_{3}$-mediated outside-in signaling during shear-flow-induced platelet mechanotransduction [25], and collaborates in collageninduced platelet responses through integrin $\alpha_{2} \beta_{1}$ [17]. Recently, cooperation between ADAP and two $\beta$-integrin cytoplasmic tail-binding proteins - talin and kindlin-3 - was revealed [26].

ADAP-knockout mice have normal growth and fertility, reduced numbers of splenic T cells and thymocytes, enlarged spleens, normal hematopoietic cellularity, moderate thrombocytopenia and normal bleeding times, but increased rebleeding from tail wounds [16, $17,27]$. Although only moderate thrombocytopenia has been reported in ADAP-knockout mice, remarkable thrombocytopenia was seen in our patients, showing a more severe phenotype in humans than predicted by the mouse model.

In ADAP-deficient mice, platelet activation, fibrinogen binding and aggregation were decreased and reduced platelet-filopodium formation was observed [16, 17, 25]. Preactivation of patients' platelets was shown by overexpression of P-selectin and PAC-1, indicating granule secretion and a conformational change in integrin $\alpha_{\mathrm{IIb}} \beta_{3}$; conversely, discoid/resting platelets with reduced pseudopodia and platelets trapped between the fibrin fibers after thrombin addition were seen on SEM. We propose that ADAP-deficient platelets, following integrin stimulation, are unable to induce intracellular signaling events that typically result in cytoskeletal and morphologic changes. These findings are supported by previous studies proposing that ADAP links integrins to actin-cytoskeleton events [16, 23, 24]. Further studies are needed to evaluate platelet function and morphology in these patients.

In ADAP-knockout mice, only moderate thrombocytopenia has been reported, with increased spleen size being postulated as its cause [17]. In the described family, no spleen enlargement or platelet antibodies were found, thus excluding those mechanisms in the pathogenesis of thrombocytopenia. We hypothesized a combination of increased clearance of activated platelets and decreased platelet production from ADAP-deficient megakaryocytes, caused by actin dysregulation and abnormal cytoskeletal function. Interestingly, a mutation in integrin $\alpha_{\mathrm{IIb}} \beta_{3}$ that changes its structure to a constitutively active one resulted in macrothrombocytopenia, suggesting impaired proplatelet formation resulting from integrin $\alpha_{\mathrm{IIb}} \beta_{3}$ 's active conformation [11].

Following submission of the present article, a second case of a family with CARST, probably because of a homozygous pathogenic variant of $F Y B$, was reported by Hamamy et al. and submitted to BMC Medical Genetics 2014, reinforcing the genetic findings reported herein.

In summary, we identified a c.393G > A (p.W131X) mutation in FYB in five homozygous individuals with congenital small-platelet thrombocytopenia and an increased bleeding tendency. Further studies are required to exclude subclinical immunologic abnormalities in this family and to better understand the molecular mechanisms involved in this disease, particularly that underlying the thrombocytopenia, and to elucidate the precise function of ADAP in different human hematopoietic cells. 


\section{Addendum}

C. Levin performed the clinical identification evaluation and patient follow-up, the research, and the data analysis, and wrote the manuscript. A. Koren performed the clinical identification evaluation and patient follow-up, and supervised the writing. E. Pretorius performed the electron microscopy research and analysis, and wrote the corresponding sections. N. Rosenberg designed the platelet-activation studies, analyzed the data, and wrote the corresponding sections. B. Shenkman performed the CPA studies and analyzed the corresponding data. H. Hauschner performed the flow-cytometry studies and analyzed the corresponding data. L. Zalman performed the hematologic laboratory analyses. M. Khayat performed the genetic analyses and wrote up the genetic results. I. Salama performed the clinical identification evaluation and patient follow-up. O. Elpeleg performed the genetic analyses, analyzed the data, and wrote up the genetic results. S. Shalev designed the genetic approach, performed the genetic analyses, analyzed the data, wrote the genetic section of the article, and supervised the writing.

\section{Acknowledgements}

This work was supported by a research grant from the Academic and Research Committee of Emek Medical Center.

\section{Disclosure of Conflict of Interests}

The authors state that they have no conflict of interest.

\section{References}

1. Drachman JG. Inherited thrombocytopenia: when a low platelet count does not mean ITP. Blood 2004; 103: 390-8.

2. Lambert MP. What to do when you suspect an inherited platelet disorder. Hematology Am Soc Hematol Educ Program 2011; 2011: 377-83.

3. Geddis AE. A POTEntial new gene for thrombocytopenia. Blood 2011; 117: 6406-8.

4. Balduini CL, Savoia A. Genetics of familial forms of thrombocytopenia. Hum Genet 2012; 131: $1821-32$.

5. Levin C, Zalman L, Tamary H, Krasnov T, Khayat M, Shalev S, Salama I, Koren A. Small-platelet thrombocytopenia in a family with autosomal recessive inheritance pattern. Pediatr Blood Cancer 2013; 60: E128-30.

6. Thrasher AJ. New insights into the biology of Wiskott-Aldrich syndrome (WAS). Hematology Am Soc Hematol Educ Program 2009; 2009: 132-8.

7. Zlotogora J. Genetics and genomic medicine in Israel. Mol Genet Genomic Med 2014; 2: $85-94$. 
8. Edvardson S, Cinnamon Y, Jalas C, Shaag A, Maayan C, Axelrod FB, Elpeleg O. Hereditary sensory autonomic neuropathy caused by a mutation in dystonin. Ann Neurol 2012; 71: 569-72.

9. Strauss T, Rozenzweig N, Rosenberg N, Shenkman B, Livnat T, Morag I, Fruchtman Y, Martinowitz U, Kenet G. Surfactant impairs coagulation in-vitro: a risk factor for pulmonary hemorrhage? Thromb Res 2013; 132: 599-603.

10. Misgav M, Shenkman B, Budnik I, Einav Y, Martinowitz U. Differential roles of fibrinogen and von Willebrand factor on clot formation and platelet adhesion in reconstituted and immune thrombocytopenia. Anesth Analg 2011; 112: 1034-40.

11. Hauschner H, Mor-Cohen R, Messineo S, Mansour W, Seligsohn U, Savoia A, Rosenberg N. Abnormal cytoplasmic extensions associated with active allbb3 are probably the cause for macrothrombocytopenia in Glanzmann thrombasthenia-like syndrome. Blood Coagul Fibrinolysis 2015; 26: 302-8.

12. Gasparyan AY, Ayvazyan L, Pretorius E, Kitas GD. Platelets in rheumatic diseases: friend or foe? Curr Pharm Des 2014; 20: 552-66.

13. Pretorius E. The role of platelet and fibrin ultrastructure in identifying disease patterns. Pathophysiol Haemost Thromb 2008; 36: 251-8.

14. Pretorius E, Briedenhann S, Marx J, Franz RC. Structural changes in the fibrin network of a Pretoria family with dysfibrinogenemia: a scanning electron microscopical study. Ultrastruct Pathol 2006; 30: 167-76.

15. Pretorius E, Du Plooy J, Soma P, Gasparyan AY. An ultrastructural analysis of platelets, erythrocytes, white blood cells, and fibrin network in systemic lupus erythematosus.

Rheumatol Int 2014; 34: 1005-9.

16. Kasirer-Friede A, Moran B, Nagrampa-Orje J, Swanson K, Ruggeri AM, Schraven B, Neel BG, Koretzky G, Shattil SJ. ADAP is required for normal alphaIIbbeta3 activation by VWF/GP Ib-IX-V and other agonists. Blood 2007; 109: 1018-25.

17. Jarvis GE, Bihan D, Hamaia S, Pugh N, Ghevaert CJ, Pearce AC, Hughes CE, Watson SP, Ware J, Rudd CE, Farndale RW. A role for adhesion and degranulation-promoting adapter protein in collagen-induced platelet activation mediated via integrin alpha 2 beta 1 . J Thromb Haemost 2012; 10: 268-77.

18. Shenkman B, Savion N, Dardik R, Tamarin I, Varon D. Testing of platelet deposition on polystyrene surface under flow conditions by the cone and plate(let) analyzer: role of platelet activation, fibrinogen and von Willebrand factor. Thromb Res 2000; 99: 353-61.

19. Togni M, Engelmann S, Reinhold D, Schraven B, Reinhold A. The adapter protein ADAP is required for selected dendritic cell functions. Cell Commun Signal 2012; 10: 14.

20. Sylvester M. Adhesion and degranulation promoting adapter protein (ADAP) is a central hub for phosphotyrosine-mediated interactions in T cells. PLoS One 2010; 5: e11708. 
21. Musci MA, Hendricks-Taylor LR, Motto DG, Paskind M, Kamens J, Turck CW, Koretzky GA. Molecular cloning of SLAP-130, and SLP-76-associated substrate of the T cell antigen receptor-stimulated protein tyrosine kinases. J Biol Chem 1997; 272: 11674-7.

22. da Silva AJ, Li Z, De Vera C, Canto E, Findell P, Rudd CE. Cloning of a novel T-cell protein FYB that binds FYN and SH2-domain-containing leukocyte protein 76 and modulates interleukin 2 production. Proc Natl Acad Sci USA 1997; 94: 7493-8.

23. Krause M. Fyn-binding protein (Fyb)/SLP-76-associated protein (SLAP), Ena/vasodilator-stimulated phosphoprotein (VASP) proteins and the Arp2/3 complex link T cell receptor (TCR) signaling to the actin cytoskeleton. J Cell Biol 2000; 149: 181-94.

24. Pauker MH, Reicher B, Fried S, Perl O, Barda-Saad M. Functional cooperation between the proteins Nck and ADAP is fundamental for actin reorganization. Mol Cell Biol 2011; 31: 2653-66.

25. Kasirer-Friede A, Ruggeri ZM, Shattil SJ. Role for ADAP in shear flow-induced platelet mechanotransduction. Blood 2010; 115: 2274-82.

26. Kasirer-Friede A, Kang J, Kahner B, Ye F, Ginsberg MH, Shattil SJ. ADAP interactions with talin and kindlin promote platelet integrin $\alpha \operatorname{Ilb} \beta 3$ activation and stable fibrinogen binding. Blood 2014; 123: 3156-65.

27. Peterson EJ, Woods ML, Dmowski SA, Derimanov G, Jordan MS, Wu JN, Myung PS, Liu QH, Pribila JT, Freedman BD, Shimizu Y, Koretzky GA. Coupling of the TCR to integrin activation by Slap-130/Fyb. Science 2001; 293: 2263-5. 